\title{
Nonstationary weak signal detection based on normalization stochastic resonance with varying parameters
}

\author{
HAIBIN ZHANG*, WEI XIONG, SHANGBIN ZHANG, QINGBO HE and FANRANG KONG \\ Department of Precision Machinery and Precision Instrumentation, University of Science and Technology of \\ China, Hefei 230026, Anhui, People's Republic of China \\ e-mail: zhbzhbyr@gmail.com
}

MS received 20 June 2015; revised 8 January 2016; accepted 8 January 2016

\begin{abstract}
The nonlinear stochastic resonance system possesses the ability of taking advantage of background noise to enhance the weak signal. It provides a new approach to detect the weak signal embedded with heavy noise. This study proposes a new varying parameter stochastic resonance employing the fourth-order RungeKutta numerical method as well as the normalized transformation of a bistable stochastic resonance system. The model performs well in the detection of a time-varying signal with background noise for denoising and signal recovery. We take the fitness coefficient and cross-correlation coefficient as the criteria and analyze the influence of different parameters. The simulating results indicate its availability, validity and that it generates a better performance than the traditional stochastic resonance. The method develops the area of time-varying signal detection with stochastic resonance and presents new strategy for detection and denoising of a time-varying signal. It can be expected to be widely used in the areas of aperiodic signal processing, radar communication, etc.
\end{abstract}

Keywords. Time-varying signal; weak signal detection; varying parameters; stochastic resonance.

\section{Introduction}

In general view, noise is always regarded as disgusted stuff in signal processing or other areas for the interference of noise to the significant components. But the nonlinear theory and experiment in past two decades indicated that to some nonlinear system, proper amount of noise can help to improve the system output signal-to-noise ratio (SNR) on the contrary. This phenomenon is called the stochastic resonance (SR) [1, 2]. According to the SR theory, when the weak signal embedded in heavy background noise is sent into some nonlinear system, the noise energy might transmit to the signal with the synergetic effect of noise and improve the system output SNR [2-4]. As the output SNR firstly increases and then decreases with the noise enhancing and a peak appears with a certain noise intensity, which conforms to the resonance phenomenon in the mechanics, it is named the SR for its nonlinear effect. For the feature of improving the output SNR, the system is applied to weak signal detection [5-9], machine fault diagnosis [10-13], energy harvesting [14-16] and some other areas obtaining abundant achievements.

In the detection of weak signal, it is different from the other methods which focus on weakening the energy of annoying noise, where SR can take advantage of the noise

*For correspondence component and whereby improve the characteristics of signal. Among the existing research, Jung and Hanggi [6] firstly presented a careful numerical study of the SR effect, without restrictions on the modulation amplitude and frequency. He has discovered a secondary resonance at smaller value of the noise strength. Zozor and Amblard [9] studied the detection of sine signal with the use of SR. As a result of the non-intuitive effect, the use of SR can be envisaged to detect small amplitude sine signal corrupted by additive noise and the authors recalled some basics of detection and revealed the detection essence. Leng et al [7] then utilized the SR to the signal detection of engineering and Chen and Varshney [5] examined the mechanism of SR effect for a fixed detector with his co-workers. In literature [8], an underdamped step-varying second-order SR method was proposed to further improve the output SNR.

The majority of research is focused on a periodic driving signal currently, which means the system input is composed of a periodic signal and random noise. Whereas we are frequently faced with some aperiodic signal in the practical application such as the linear frequency modulated (LFM) signal, nonlinear frequency modulated (NLFM) signal, Doppler signal and so on [17, 18]. All the signals widely exist in areas of wireless communication, radar technique, acoustic signal processing and some others. Aperiodic signal can also suffer from the noise and get unfavorable to detect and identify $[17,19]$. Some researchers also took 
advantage of SR and much effort were concentrated on aperiodic stochastic resonance (ASR) [20, 21] including the aperiodic force [22], chaotic input [23], shock signal [24], etc. Considering all the research, they just studied the performance of traditional SR under condition of an aperiodic input, which means the model was the same with the periodic one. For the SNR in fractional domain in literature [25], it can be only used in the LFM signal as the signal energy still concentrates in a certain direction while it is impossible for some NLFM signal. In fact, the traditional SR model cannot always match an aperiodic input, because the fixed parameters can only be effective to a certain driving frequency while the frequency of aperiodic input varies with time going. Furthermore SR is still restrained by the adiabatic approximate conditions and the input signal should meet the small parameter requirement. This restricts the practical application of the system yet.

To overcome all the issues, a new varying parameters and normalization SR (VPNSR) system based on the normalized transformation [26] is proposed with numerical calculation by the discrete fourth-rank Runge-Kutta method [27]. System parameters are firstly converted for normalization in the model to satisfy the small parameter requirement. The coefficient of fitness (R-square, $R^{2}$ ) and its correlation coefficient of output signal are regarded as the criteria of the system performance. Varying parameters coinciding with frequency of input signal are obtained and applied to determine the parameters of VPNSR. Finally the optimal parameters are searched and the optimized output signal acts as the final recovered signal with noise eliminated effectively. The rest of the paper is arranged as follows. Section 2 shows the basic theory of SR and its normalization. The proposed VPNSR and its output criteria are described in section 3. In section 4, two different kinds of frequency modulated signals including LFM and varying speed Doppler signal are employed to verify the availability of VPNSR in signal denoising and recovery. Its performance is analyzed in section 5. Finally some conclusions and prospects are drawn in section 6 .

\section{Bistable stochastic resonance and its normalization}

\subsection{Bistable SR system}

The three basic ingredients of producing SR phenomenon are as follows: [1] a bistable or multi-stable system, [5] a weak coherent input (such as a periodic signal), and [20] a source of noise that is inherent in the system, or that adds to the coherent input. Considering the overdamped motion of a Brownian particle in a bistable potential in the presence of noise and periodic forcing, an SR system can be expressed as [2]:

$$
\frac{\mathrm{d} x}{\mathrm{~d} t}=-V^{\prime}(x)+s_{\mathrm{m}}(t)=-V^{\prime}(x)+s(t)+n(t) .
$$

Considering the common form of a bistable SR system, $V(x)$ in the formula stands for the reflection-symmetric quartic potential with bistable state which can be denoted in the form of $V(x)=-\frac{a}{2} x^{2}+\frac{b}{4} x^{4} . a$ and $b$ are real parameters. $s_{\mathrm{m}}(t)$ is the mixed input signal composed of regular driving signal $s(t)$ (generally a periodic signal but a varying signal in this paper) and $n(t)=\sqrt{2 D} \xi(t)$ with $n(t) n(t+\tau)=2 D \delta(\tau)$ where $D$ is the noise intensity and $\xi(t)$ presents a zero-mean, unit variance Gaussian white noise. As the system is utilized to deal with the timevarying signal here, $s(t)$ has the expression of $s(t)=A \cos \left(2 \pi\left(\int f(t) \mathrm{d} t\right)\right)$. Substituting it into Eq. (1), the expression with a common form is denoted as

$$
\frac{\mathrm{d} x}{\mathrm{~d} t}=a x-b x^{3}+A \cos \left(2 \pi\left(\int f(t) \mathrm{d} t\right)\right)+n(t) .
$$

In the formula, $A$ indicates the signal amplitude and $f(t)$ shows the varying frequency. When the LFM signal acts as the driving signal, $f(t)$ stands in the form of $\left(f_{0}+k t\right)$. According to Eq. (2), there will be two potential wells of doubly degenerate minima locating at $x_{\min }= \pm \sqrt{a / b}$. The height of the potential barrier between the minima is given by $\Delta V=a^{2} / 4 b$. When $A>0$ and the driving signal is periodic, the input will bring a periodic transition of the potential wells. If the system, driving signal and noise reach to some matching relation, the noise will synchronize to the signal and they work on the system jointly. In this case, the weak input signal $s(t)$ gets highly enhanced by the noise and a phenomenon like the resonance coming up. This is the effect of SR. But to weak aperiodic signal, the matching relation cannot be reached all the time with fixed system parameters. In order to overcome the difficulty and develop the application of SR in time-varying signal detection, a new VPNSR is proposed.

\subsection{Normalization of bistable SR}

To the bistable SR system shown as Eq. (1), many researchers [2, 4] have paid lots of effort. They set up the approximate analytical theory under adiabatic approximation assumption [2]. Adiabatic approximation condition mainly assumes that the driving frequency is much less than the Kramers rate (KR):

$$
r_{k}=\frac{a}{\sqrt{2}} \exp \left(-\frac{\Delta V}{D}\right)
$$

and the amplitude of driving signal as well as the noise intensity are less than 1 , which is called the small parameter limitation [28]. So in practical application, the parameters step over the limitation easily and the adiabatic approximation condition cannot be satisfied. When solving the equation directly, SR effect will not occur and the signal cannot be detected successfully. This is on account of that 
too high driving frequency leads to the lag of system response to the input and larger signal amplitude is essential to drive the system for the occurring of SR. But the actual signal does not meet the requirement. In order to get over the small parameter restriction, Leng et al [28] proposed the re-scaling frequency stochastic resonance and $\mathrm{Hu}$ et al [26] developed the normalization theory with his coworkers. Re-scaling frequency converts the high frequency signal into a new low one meeting the small parameter limitation by a conversion scale $r$. Then its characteristic frequency is identified in the output signal with the new frequency. Finally the original signal with high frequency and noise eliminated is regained by the scale $r$ again. While the optimal re-scaling rate needs to be found and the numerical simulation is easy to be divergent when the input SNR is fixed with large signal amplitude. This makes it difficult for us to accomplish the optimization procedure. Therefore the normalization strategy is taken to realize the large signal parameters (mainly referring to the driving frequency) SR with time-varying system parameters. When the parameters $a$ and $b$ in Eq. (2) are not equal to 1, consider the transformation:

$$
\left\{\begin{array}{l}
y=x \sqrt{\frac{b}{a}} \\
\tau=a t
\end{array}\right.
$$

Substituting it into Eq. (2), we get

$$
\begin{aligned}
a \sqrt{\frac{a}{b}} \frac{\mathrm{d} y}{\mathrm{~d} \tau}= & a \sqrt{\frac{a}{b}} y-a \sqrt{\frac{a}{b}} y^{3}+A \cos \left(2 \pi\left(\int f\left(\frac{\tau}{a}\right) \mathrm{d} \frac{\tau}{a}\right)\right) \\
& +n\left(\frac{\tau}{a}\right) .
\end{aligned}
$$

After the transformation, the noise in Eq. (5) is represented by $n\left(\frac{\tau}{a}\right)$. While $n(t)$ means the Gauss white noise which distributes uniformly in frequency domain. $n\left(\frac{\tau}{a}\right)$ still indicates the zero-mean white noise with intensity of $D$. Simplifying the expression, there is the expression:

$$
\frac{\mathrm{d} y}{\mathrm{~d} \tau}=y-y^{3}+\sqrt{\frac{b}{a^{3}}} A \cos \left(2 \pi\left(\int f\left(\frac{\tau}{a}\right) \mathrm{d} \frac{\tau}{a}\right)\right)+\sqrt{\frac{b}{a^{3}}} n\left(\frac{\tau}{a}\right) .
$$

Comparing Eq. (2) and Eq. (6), the system parameters are both altered into 1 which means the realization of normalization. Meanwhile the characteristic frequency of input signal changes from $f(t)$ to $\frac{1}{a} f\left(\frac{\tau}{a}\right)=\frac{1}{a} f(t)$. When $a$ is a large rational number, the driving frequency in Eq. (6) is extremely reduced, which cover the small parameter restriction. The amplitude of signal and noise are amplified $\sqrt{\frac{b}{a^{3}}}$ fold at the same time. This suggests us that for input signal with high characteristic frequency, the reduction of the driving frequency is realized by choosing a large system parameter $a$ and amplifying the mixed input signal $\sqrt{\frac{a^{3}}{b}}$ fold, because the two equations are totally equivalent. In this case, parameters $b$ acts the role of controlling the amplitude of output signal. After the normalization, we take the numerical solution for the typical first-order differential equation as Eq. (2). The discrete fourth-rank Runge-Kutta method [27] as follows is applied to solve the equation numerically.

$$
\left\{\begin{array}{l}
x^{\prime}=\frac{\mathrm{d} x}{\mathrm{~d} t}=f(t, x), x(t[0])=x[0] \\
k_{1}=f(t[n], x[n]) \\
k_{2}=f\left(t[n]+\frac{h}{2}, x[n]+\frac{h}{2} k_{1}\right) \\
k_{3}=f\left(t[n]+\frac{h}{2}, x[n]+\frac{h}{2} k_{2}\right) \\
k_{4}=f\left(t[n]+h, x[n]+h k_{3}\right) \\
x[n+1]=x[n]+\frac{h}{6}\left(k_{1}+k_{2}+k_{3}+k_{4}\right)
\end{array} .\right.
$$

\section{VPNSR and its output criteria}

The SR system acts as a narrow-band filter in fact. In traditional SR system, it is essential that the period of input, noise intensity and system parameters should satisfy the certain matching condition. When half of the transition rate as Eq. (3) matches the signal frequency, the SR might come up. But for aperiodic signal whose frequency varies with time, the matching condition cannot be met all the time. In last section, it has been mentioned that in the procedure of normalization, parameter $a$ plays the crucial role for the large parameter while $b$ just affects the output amplitude, which means the location of potential well. According to the expression of $r_{k}$ as Eq. (3), when the signal amplitude is $\sqrt{\frac{a^{3}}{b}}$-fold amplified, the noise intensity change to $D^{\prime}=\frac{a^{3}}{b} D$. Substituting $\Delta \mathrm{V}$ and $D^{\prime}$ into Eq. (3) we get the new Kramers rate:

$$
r_{k}^{\prime}=\frac{a}{\sqrt{2}} \exp \left(-\frac{a^{2} / 4 b}{a^{3} D / b}\right)=\frac{a}{\sqrt{2}} \exp \left(-\frac{1}{4 a D}\right)
$$

When $a$ is a rational number large enough, $\exp \left(-\frac{1}{4 a D}\right) \approx 1$. So $r_{k}$ after normalization is in direct proportion to $a$. This provides us a much higher Kramers rate which demonstrates the possibility of SR effect on the other hand. On this ground, a new varying parameter SR system is proposed to detect the ascertained type time-varying signal. In the system, parameter $a$ varies synchronously with the driving frequency and is denoted by $a(t)$. Under the circumstances, the new parameter $a(t)$ can make the driving frequency of the new SR system constant relatively after the normalization and the matching condition will be established at different time. So for the system of Eq. (2), the new SR system guarantee the occurrence of SR effect with expression as 


$$
\begin{aligned}
\frac{\mathrm{d} x}{\mathrm{~d} t}= & a(t) x-b(\mathrm{t}) x^{3}+A \cos \left(2 \pi\left(\int f(t) \mathrm{d} t\right)\right)+n(t) \\
= & \left(\frac{a_{0} f(t)}{f(0)}\right) x-\left(\frac{b_{0} f(t)}{f(0)}\right) x^{3} \\
& +A \cos \left(2 \pi\left(\int f(t) \mathrm{d} t\right)\right)+n(t)
\end{aligned}
$$

where $a(t)=\frac{a_{0} f(t)}{f(0)}$ and it can result in a new driving frequency for SR system after normalization according to Eq. (6) with result of

$$
f^{\prime}(\tau)=\frac{f(t)}{a(t)}=\frac{f(0)}{a_{0}}=\text { const } .
$$

Equation [14] indicates the proposed VPNSR. Its driving frequency keeps constant to establish the matching condition. Meanwhile, we tend to maintain the balance position at $x_{\min }= \pm \sqrt{\frac{a}{b}}$ to make the consistency of the output signal. So set the other parameter $b(t)=\frac{b_{0} f(t)}{f(0)}$. After amplifying the input signal with high characteristic frequency $\sqrt{\frac{a(t)^{3}}{b(t)}}$-fold and solve Eq. (9) numerically, the output signal of VPNSR with noise reduced is obtained and original signal is recovered successfully.

But the most important issue is how to determine the parameters $a(t)$ and $b(t)$. Here the original signal type is needed, which means the LFM or the Doppler signal but the specific arguments are unknown. Besides, the initial parameters like $a_{0}$ and $b_{0}$ need to be optimized to gain the best output. All the tasks cannot be accomplished without the evaluation indicator for the output. In fact, there will not be a distinct spectral line in its frequency spectrum of Fourier transformation to a time-varying signal. The SNR [26, 28] in traditional SR system as the indicator will not work here. In the research of aperiodic SR, many researchers [21, 29] tend to take the maximal cross-correlation coefficient of the pure signal $s(t)$ in input and the output signal $x(t)$ denoted by $C_{s x}$ here. For the discrete signal, it can be expressed as

$$
C_{s x}=\max \left\{\frac{\sum_{j=1}^{N}[s(j+\tau)-\bar{s}][x(j)-\bar{x}]}{\sqrt{\sum_{j=1}^{N}[s(j)-\bar{s}]^{2} \sum_{j=1}^{N}[x(j)-\bar{x}]^{2}}}\right\}_{\tau} .
$$

In the formula, $\tau \in\left[-f_{s} \cdot T_{\max } / 2, f_{s} \cdot T_{\max } / 2\right]$ where $f_{s}$ means the sampling frequency and $T_{\max }$ indicates the maximal period of original signal. Operator max means the maximal value for all the variable $\tau$. $N$ is the length of signal and $\bar{s}=\frac{1}{N} \sum_{j=1}^{N} s(j), \bar{x}=\frac{1}{N} \sum_{j=1}^{N} x(j)$. It should be noticed that for calculating the indicator, the pure signal without noise is essential, which is scarcely possible for us in practical application. So another indicator is considered during our study which is the fitness coefficient (R-square, $R^{2}$ ) in statistical category.

$$
R^{2}=1-\frac{S S E}{S S T}=1-\frac{\sum_{i=1}^{N}(x(i)-\widehat{x(i)})^{2}}{\sum_{i=1}^{N}(x(i)-\bar{x})^{2}} .
$$

In the above equation, SSE means the error quadratic sum of the real value and fitting value and SST indicates the error quadratic sum of the real value and the mean value. $x(i)$ and $\bar{x}$ represent the SR output and its mean value separately. $\widehat{x(i)}$ is the fitting result of the output signal with certain signal type. It is apparent that $R^{2} \leq 1$ and a higher value means that the output signal is more consistent with the fitting result, which means the fitting error is smaller and the system generates a better performance.

\section{Detection and recovery of two different kinds of signal}

\subsection{LFM signal}

The LFM signal is the one whose frequency is linearly modulated. So we denote its instantaneous frequency as $f(t)=f_{0}+k t$ where $f_{0}$ means the initial frequency and $k$ is the modulated rate. Substitute the $f(t)$ into signal $s(t)=A \cos \left(2 \pi\left(\int f(t) \mathrm{d} t\right)\right)$ :

$$
s(t)=A \cos \left(2 \pi\left(f_{0} t+\frac{k}{2} t^{2}\right)+\varphi\right)
$$

Since the signal type is foregone, the time-varying parameters $a(t)$ and $b(t)$ in Eq. (9) have the certain expression with some specific arguments to be decided.

4.1a Output with presetting parameters: In order to study the output performance of VPNSR, a simulation signal is employed. According to the above description, Matlab is utilized to take the numerical simulation analysis. Firstly a simulated signal as Eq. (13) is created which contains modulated rate $k=10,000 \mathrm{~Hz} / \mathrm{s}$, initial frequency $f_{0}=100 \mathrm{~Hz}$, initial phase $\varphi=0$ and signal amplitude $A=1$. Assuming that the sampling frequency $f_{s}=200 \mathrm{kHz}$ and sampling time $T=0.1 \mathrm{~s}$. The sampling frequency $\mathrm{f}_{\mathrm{s}}$ during our simulation work is selected freely, which meets the Nyquist law in the whole frequency band. As the data length is not such enormous that a relative high sampling frequency is permissible. So it is set at $200 \mathrm{kHz}$ in the whole cases of this paper. The pure original signal is then shown as the red curve in the left oscillogram of figure 1(a). Noise with SNR of $-3 \mathrm{~dB}$ is injected by the function awgn. The mixed signal with its waveform is shown by the black line. Here an intuitive analysis is made in the time domain, so the noise added is not such severe that we can tell the distinct component in its spectrum of figure 1(b). This will not affect the comparison between the traditional SR and the new VPNSR and it also allows us to have a more clear comprehension by the waveform on the contrary. In its 

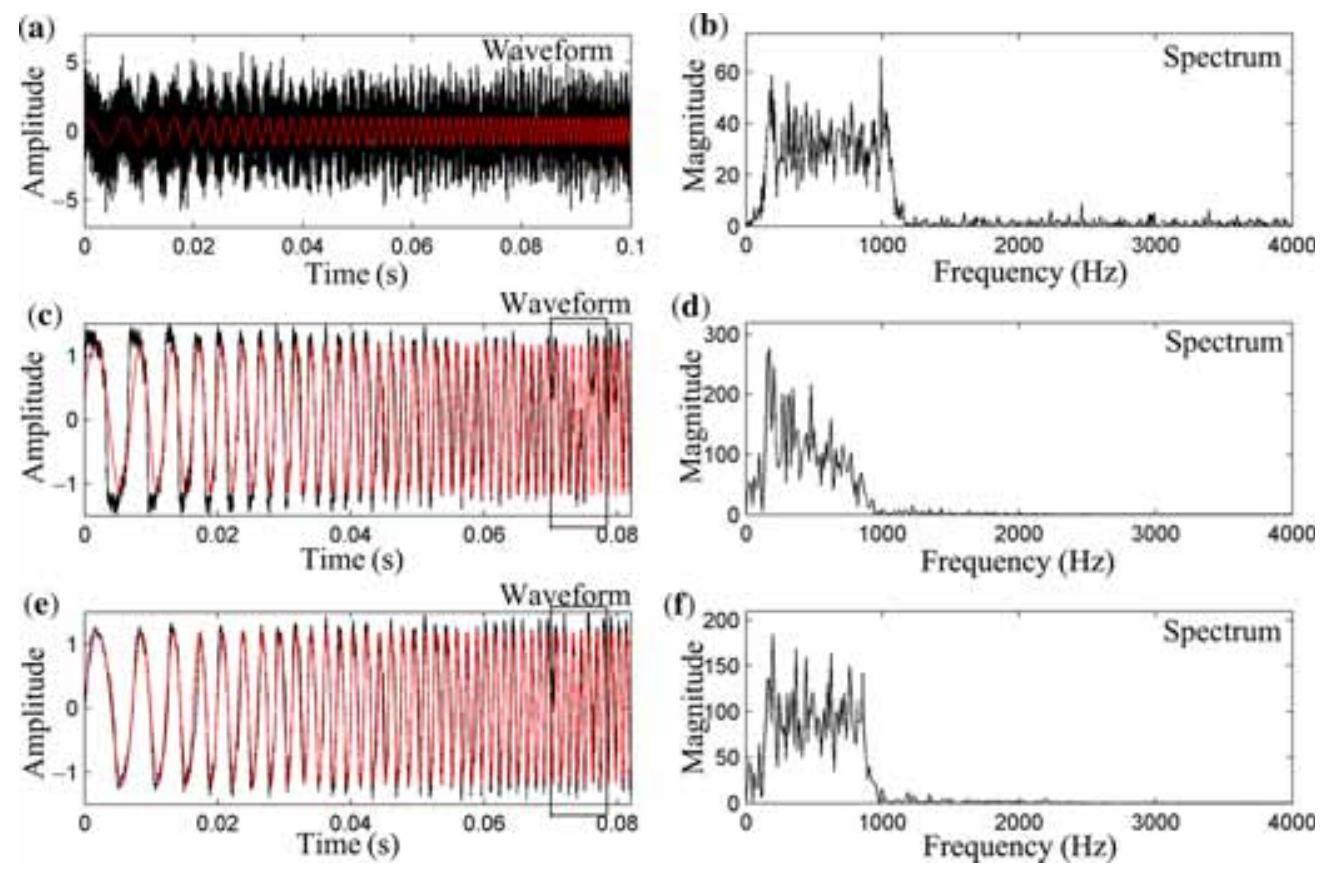

Figure 1. LFM input signal with noise and its output of two systems, (a) original input signal with noise of $-3 \mathrm{~dB}$, (b) spectrum of input signal, (c) output signal of traditional SR, (d) spectrum of traditional SR output, (e) output signal of VPNSR, and (f) spectrum of VPNSR output.

oscillogram, the LFM signal is found to be totally submerged in noise and almost impossible to identify.

Since the input signal is ready, the two systems with presetting parameters are taken to dispose the mixed signal so a direct impression on the system performance is presented. To the traditional SR system, set parameters $a=b=6000$, which will make an average new frequency of

$$
((100 \mathrm{~Hz}+10,000 \mathrm{~Hz} / \mathrm{s} \times 0.1 \mathrm{~s})+100 \mathrm{~Hz}) / 2 / a=0.1 \mathrm{~Hz} .
$$

Amplifying the amplitude of input signal by $\sqrt{\frac{a^{3}}{b}}=6000$ fold and substituting it into Eq. (2), we obtain the output signal shown as the black curve figure 1(c) after solving it with Eq. (7). Take the lsqcurvefit function in Matlab to make the curve fitting (all the fitting processing is realized in the same way of this paper) with fitting function of $s^{\prime}(t)=A^{\prime} \cos \left(2 \pi\left(100 t+\frac{10,000}{2} t^{2}\right)+\varphi^{\prime}\right)$ where the $\mathrm{A}^{\prime}, \varphi^{\prime}$ mean the fitting amplitude and initial phase. For the sake of high convergence speed and simulation efficiency, fix the initial frequency at $100 \mathrm{~Hz}$ and modulated rate at 10,000 $\mathrm{Hz} / \mathrm{s}$. In practical application, the two parameters are unknown and their estimation method of our system is described later. After the curve fitting operation, we get results of $\left[\mathrm{A}^{\prime}, \varphi^{\prime}\right]=[1.1689,-1.1416 \mathrm{rad}]$ and the red line in figure 1(c) indicates the fitting curve. Calculating the two indicators introduced in section 3 , the maximal cross-correlation coefficient $C_{s x 1}=0.8667$ is found and fitness coefficient $R_{1}^{2}=0.7494$.
Follow on, to the proposed VPNSR, set the parameters $k=10,000 \frac{\mathrm{Hz}}{\mathrm{S}}, f_{0}=100 \mathrm{~Hz}, \quad a(t)=a_{0}+\frac{a_{0}}{f_{0}} k t\left(a_{0}=1000\right)$ and $b(t)=a(t)$. In this case, the driving frequency will be converted into $\frac{f(t)}{a(t)}=0.1 \mathrm{~Hz}$. Substituting the signal with amplitude amplified by $\sqrt{\frac{a(t)^{3}}{b(t)}}$-fold into Eq. (2) and solving the equation, the output signal of proposed system is shown in figure $1(\mathrm{e})$. The red line still indicates the fitting result with $\left[\mathrm{A}^{\prime}, \varphi^{\prime}\right]=[1.1874,-1.4172 \mathrm{rad}]$. The two criteria are calculated out again with $C_{s x 2}=0.9462$ and $R_{2}^{2}=0.8947$.

Some conclusions are reached by comparing the two systems outputs. In time domain of figure 1(c) and (e), the output of VPNSR contains less noise than the traditional one and is closer to the pure LFM signal especially in the band of low and high frequency. Note that in the high frequency band, the transition even disappears in the traditional SR system as in the frame of figure 1(c), but not the same for the proposed system. The different output indicators also provide the similar point that the new model had a higher criterion obviously. From the two output spectrums in figure 1(d) and (f), the high frequency band is almost eliminated by the traditional SR system while it is well retained in our model. This is easy to understand that with a fixed parameter $a$, the new frequency still varies with time and gets higher in the high frequency band. So the SR effect cannot be observed distinctly in the traditional SR. The proposed system overcomes the problem successfully by converting the new frequency to the same level. 

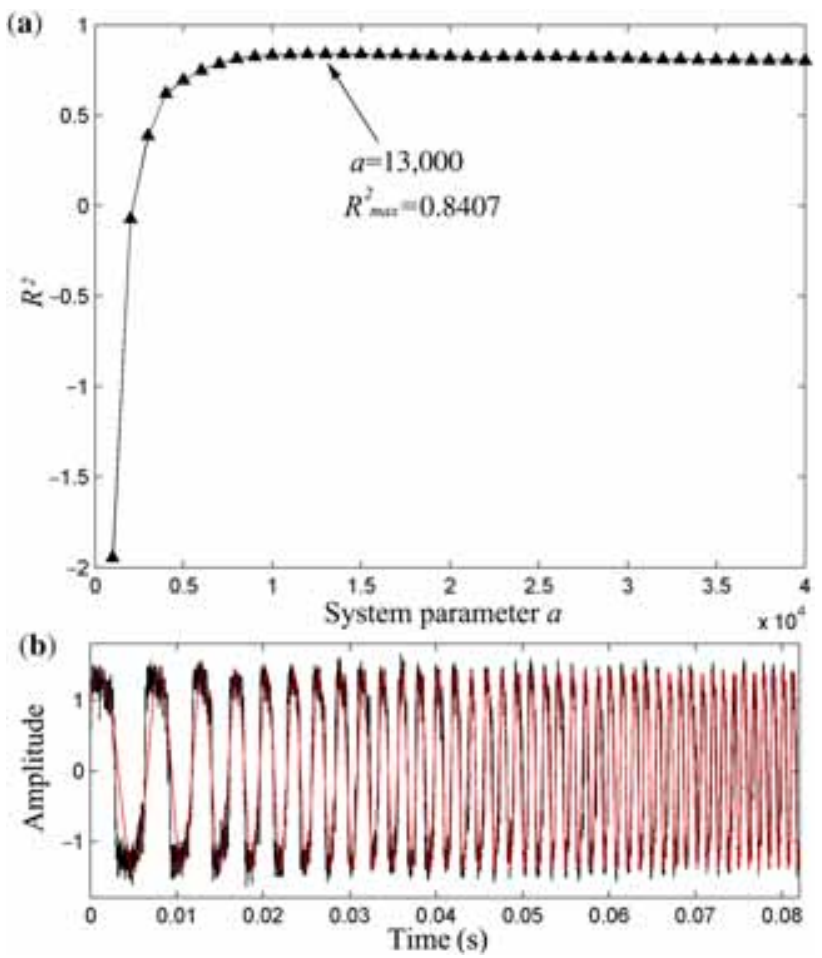

Figure 2. Optimal output of LFM signal for traditional SR, (a) different output $R^{2}$ with variation of $a$ and (b) optimal output waveform.

4.1b Output with optimal parameters: From the above analysis, all the parameters are preset and the system is not under the optimal condition. Besides the LFM signal parameters are also unknown which is treated as known quantities. Next, the signal parameters are estimated according to the input signal as well as the optimal combination of the system parameters.

For a comparison, the traditional SR system is optimized firstly and utilized it to denoise the input signal. Set the fitness coefficient $R^{2}$ as the evaluation index and calculate its value for different system parameter $a(b=a)$. Making $a$ change from 1000 to 40,000 with changing step of 1000 and work on the same signal as last section, the different results are shown in figure 2(a). The curve shows that its output $R^{2}=0.8407$ gets maximal when $a=b=13,000$ which means the system gets to the optimal condition. Then process the signal whose amplitude is 13,000-fold amplified with the optimized system and gets the output signal with waveform shown as figure 2(b). The black line indicates the original output and red curve shows the fitting result. The output signal is found to contain less noise components and closer to pure signal when compared to that in figure 1(c) with the calculated cross-correlation coefficient $C_{s x}=0.9169$.

Then take the VPNSR system to deal with the same input signal for searching of an optimal output. According to Eq. (13), the proposed model has four parameters of $a_{0}=b_{0} f_{0}$ and $k$, first two of which are system parameters and the other two are signal parameters. The optimal values of the system parameters are needed and the signal parameters for the further calculation need to be estimated. Figure 3 shows the searching results. During the searching procedure, $s^{\prime}(t)=A^{\prime} \cos \left(2 \pi\left(\hat{f}_{0} t+\frac{\hat{k}}{2} t^{2}\right)+\varphi^{\prime}\right)$ is taken to fit the output signal $x(t)$ of each set of $\hat{f}_{0}, \hat{k}$ and take the $R^{2}\left(\hat{f}_{0}, \hat{k}\right)$ as the output criterion in the first step. This is to estimate the appropriate signal parameters. In figure 3(a), fix $k=10,000 \mathrm{~Hz} / \mathrm{s}$ and $a_{0}=b_{0}=1000$, the initial frequency $f_{0}$ changes from $10 \mathrm{~Hz}$ to $400 \mathrm{~Hz}$ with step of 10 Hz. The different output $R^{2}$ for different $f_{0}$ are obtained with a peak value of $R^{2}=0.8947$ at $f_{0}=100 \mathrm{~Hz}$. Figure $3(\mathrm{~b})$ shows us the different outputs $R^{2}$ with the variation of $k$ where $f_{0}=100 \mathrm{~Hz}$ and system parameters are same as before. The modulated rate $k$ increases from $5000 \mathrm{~Hz} / \mathrm{s}$ to $10,000 \mathrm{~Hz} / \mathrm{s}$ every $200 \mathrm{~Hz} / \mathrm{s}$ and results indicate that the maximal value comes up when $k=10,000 \mathrm{~Hz} / \mathrm{s}$. Since now the estimation for signal parameters is processed successfully. In second step, the system parameters $a_{0}$ and $b_{0}$ need to be optimized as they applied before were presetting ones. In figure 3(c), fix $f_{0}=100 \mathrm{~Hz}$ and $k=10,000 \mathrm{~Hz} / \mathrm{s}$ while change $a_{0}\left(b_{0}\right)$ from 600 to 2550 with varying step of 50 . Sending every set of parameters into Eq. (9) and solving it with normalization, there are the output $R^{2}$ for different groups. It is obvious that $R^{2}=0.9081$ gets to the highest value when $a_{0}=b_{0}=1400$, where the $C_{s x}=0.9530$ is also calculated out. The $R^{2}$ gets further improved when compared to the output with presetting parameters. Solving Eq. (9) with all the optimal parameters, the output signal is shown in figure 4, where black line indicates the original signal and red one means the fitting results. Intuitively, they match to each other quite well which means the noise gets eliminated more downright and the original LFM signal gets effectively recovered with its parameters estimated successfully.

Enumerating all the simulating results into table 1 including the results of presetting parameters and optimal results for two different systems, we find that the proposed system performs much better than the traditional one. It has the superiority on signal denoising and recovery which also provides us the signal parameters. It can bring much convenience in the later signal processing and information extraction.

In figure 3, we utilized the estimation of the signal parameters and optimization of the system parameters. During the optimization, the parameter needing to be optimized is searched in a certain range while the others are fixed. This is just for a better illustration of the influence of each parameter on the output $R^{2}$. While in practical application, the optimization procedure should be achieved by conducting a three-dimensional search in the $f_{0^{-}} k-a_{0}$ space. Considering all the issues, detecting and recovery procedure of a time-varying signal with the proposed VPNSR is conducted. As the three-dimensional search is very 

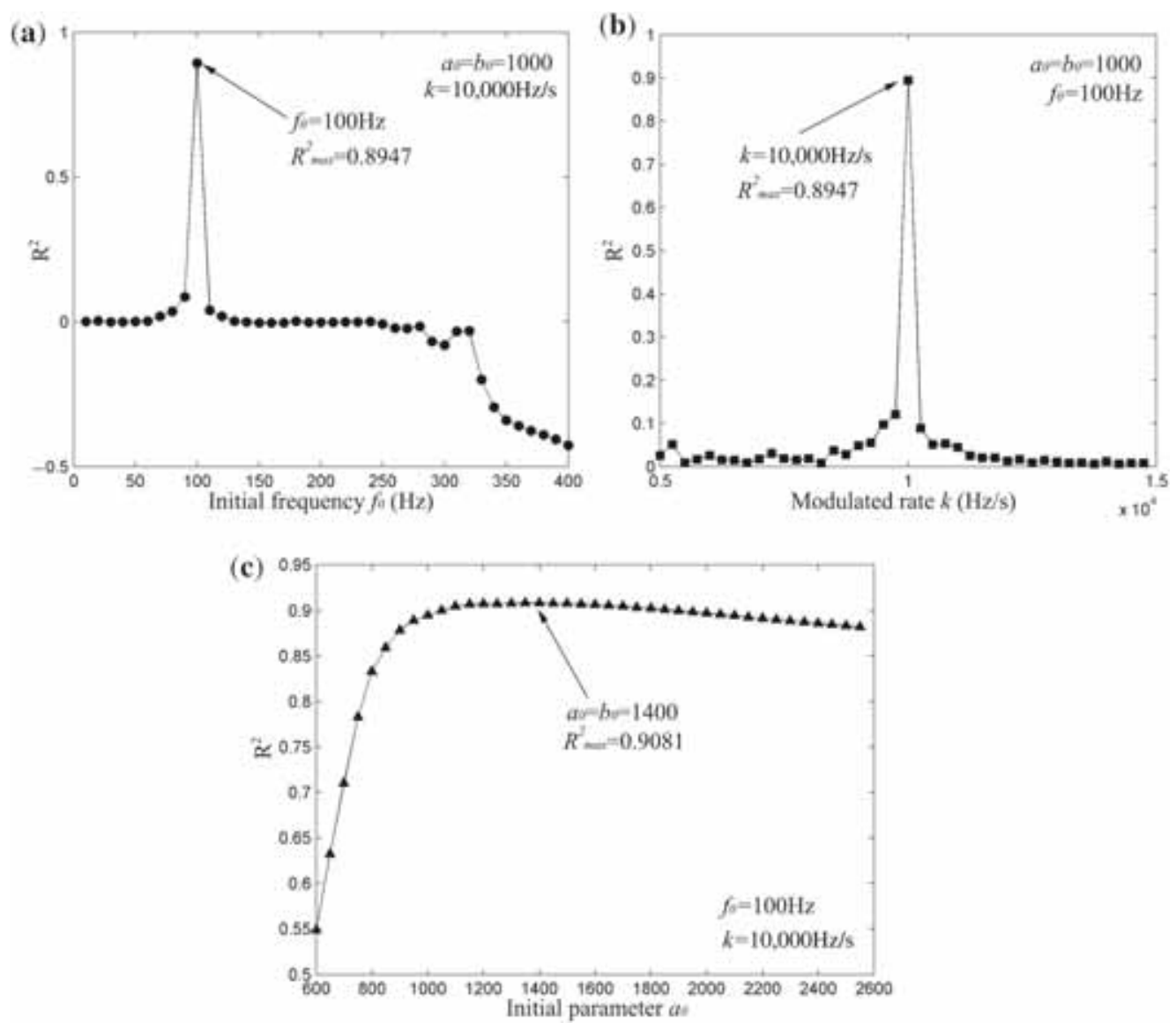

Figure 3. LFM output $R^{2}$ with variation of parameters for VPNSR, (a) $R^{2}$ with variation of initial frequency $f_{0}$, (b) $R^{2}$ with variation of modulated rate $k$, and (c) $R^{2}$ with variation of system parameter $a_{0}$.

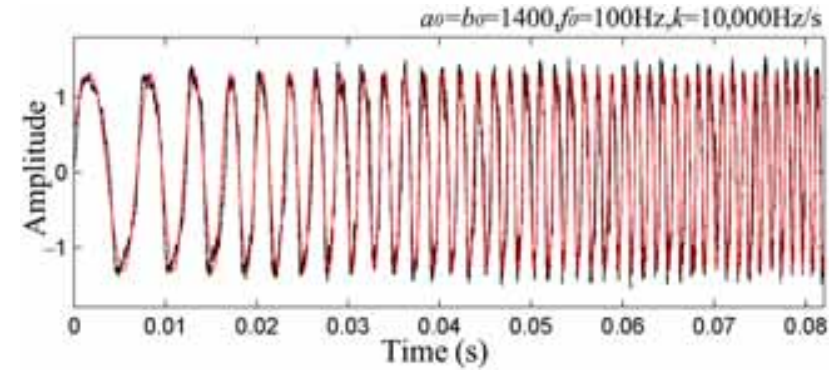

Figure 4. Optimal output waveform of LFM signal for VPNSR

Table 1. LFM signal output results of two different systems

\begin{tabular}{lccc}
\hline & & \multicolumn{2}{c}{ Output results } \\
\cline { 3 - 4 } Different systems & System parameters & $R^{2}$ & $C_{s x}$ \\
\hline Traditional SR & $a=b=6000$ & 0.7494 & 0.8667 \\
Traditional SR & $a=b=13,000$ & 0.8407 & 0.9169 \\
$\quad$ (optimized) & & & \\
VPNSR & $a_{0}=b_{0}=1000$ & 0.8947 & 0.9462 \\
VPNSR (optimized) & $a_{0}=b_{0}=1400$ & 0.9081 & 0.9530 \\
\hline
\end{tabular}

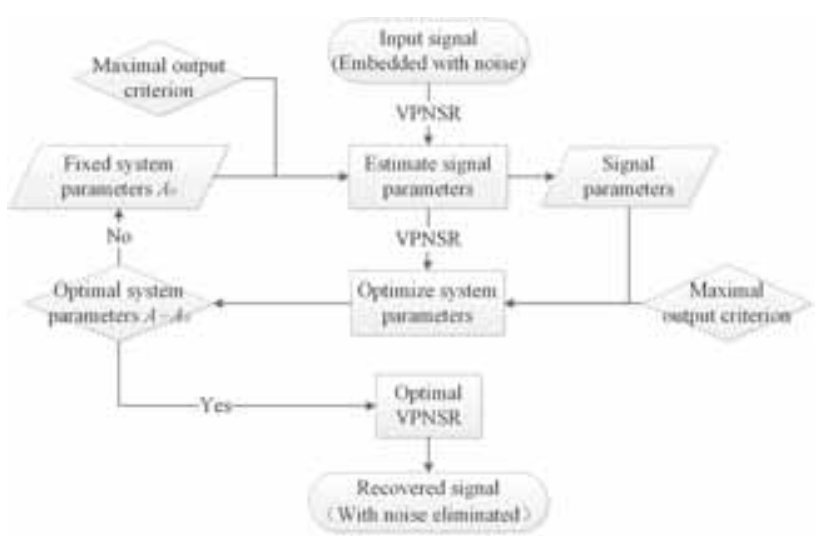

Figure 5. Time-varying signal detection and recovery scheme of VPNSR.

inefficient, it is realized by separating into two parts which are a two-dimensional search for $f_{0}, k$ and one-dimensional search for optimal system parameter $a_{0}$. Figure 5 shows the whole scheme. For a time-varying signal (not only the LFM here) with certain frequency modulated mode, we firstly send the mixed signal with strong background noise into the VPNSR system with preset system parameters. Effective 
estimation of signal parameters (like $f_{0}, k$ here) with the different output results for different signal parameters is realized. Definite peak value of the output criterion will appear on certain position where the signal parameters are what we need. Secondly, the signal model is fixed and the system parameters $a(t)$ and $b(t)$ are set according to the certain frequency modulated mode as Eq. (9). In this step, the initial system parameters $a_{0}$ and $b_{0}$ are the ones remaining unknown that need to be optimized. Calculate the different output results with a varying $a_{0}$ and the value related to the highest output criterion shows the optimal result. Then if the optimized system parameters $A=A_{0}$ (contain $a_{0}$ and $b_{0}$ here), go on with the next step or else return to first step and change the presetting system parameters from $A_{0}$ to $A$. Thirdly, substituting the two sets of parameters to Eq. (9) and solving it, there is the final recovered signal with noise eliminated which can be utilized for further research. For the other time-varying signal like Doppler or other NLFM signal, changing the system parameters model in Eq. (9) is needed to realize the detection and recovery of different signals.

\subsection{Doppler signal with acceleration}

The Doppler signal with acceleration is usually the acoustic signal acquired from an accelerated moving sound source shown as figure 6 . The frequency of sound is $f_{0}$ while it varies when it gets to the observer. On the initial position

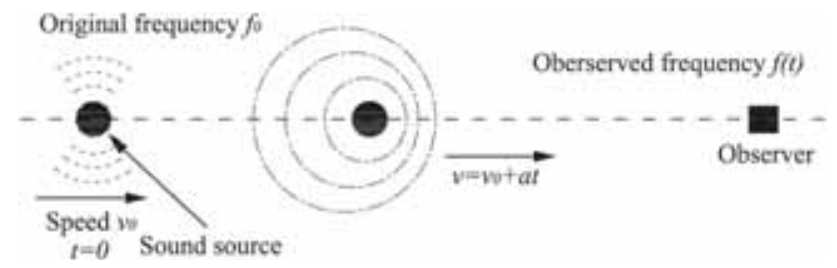

Figure 6. Geometric model for the Doppler acceleration signal. the sound source speed is denoted as $v_{0}$ when $t=0$. As a result of the acceleration $a$, the source speed gets to $v(t)=v_{0}+a t$ at time $t$. According to the acoustic theory of Morse and Ingard [30], there is the instantaneous frequency acquired by the observer:

$$
f(t)=\frac{f_{0}}{1-M(t)}=\frac{f_{0}}{1-\frac{v_{0}+a t}{c}}=\frac{c f_{0}}{c-\left(v_{0}+a t\right)}
$$

In the above formula, $M(t)$ indicates the Mach number at different time and $c=340 \mathrm{~m} / \mathrm{s}$ means the sound velocity. Then the Doppler signal from the observer can be denoted as

$$
\begin{aligned}
s(t) & =A \cos \left(2 \pi\left(\int f(t) \mathrm{d} t\right)\right) \\
& =A \cos \left(\frac{2 \pi c f_{0}}{a} \ln \left(c-v_{0}-a t\right)+\varphi\right)
\end{aligned}
$$

Since the signal type is foregone again, the time-varying parameters $a(t)$ and $b(t)$ in Eq. (9) have the certain expression where the initial speed $v_{0}$, original frequency $f_{0}$ and acceleration $a$ remain unknown. On this occasion, take the VPNSR to deal with the simulating signal directly to verify the detecting and recovery procedure as well as its availability and superiority. Firstly a simulating Doppler signal is produced with parameters of initial phase $\varphi=0$, signal amplitude $A=1, v_{0}=150 \mathrm{~m} / \mathrm{s}, f_{0}=1000 \mathrm{~Hz}$ and $a=50 \mathrm{~m} / \mathrm{s}^{2}$. The sampling frequency $f_{s}=200 \mathrm{kHz}$ and sampling time $T=1 \mathrm{~s}$. Then the mixed input signal is then shown as the waveform in figure $7 \mathrm{a}$ with noise of $\mathrm{SNR}=-3 \mathrm{~dB}$. The figure shows the detail waveform of the original signal during time interval of $[0 \mathrm{~s}, 0.01 \mathrm{~s}]$ and $[0.64 \mathrm{~s}, 0.65 \mathrm{~s}]$ from which we can hardly identify the pure Doppler signal of red line as the background noise. Calculating the $R^{2}$ and $C_{s x}$ of the input signal, there are $R^{2}=0.1973$ and $C_{s x}=0.4442$. Next, process the signal according to the scheme in figure 5 sequentially. In order to employ the proposed VPNSR, the signal parameters of $v_{0}$, $f_{0}$ and $a$ need to be estimated firstly. Similarly as the LFM signal, set the fitness coefficient $R^{2}$ as the evaluation index and calculate its value for different signal parameters with

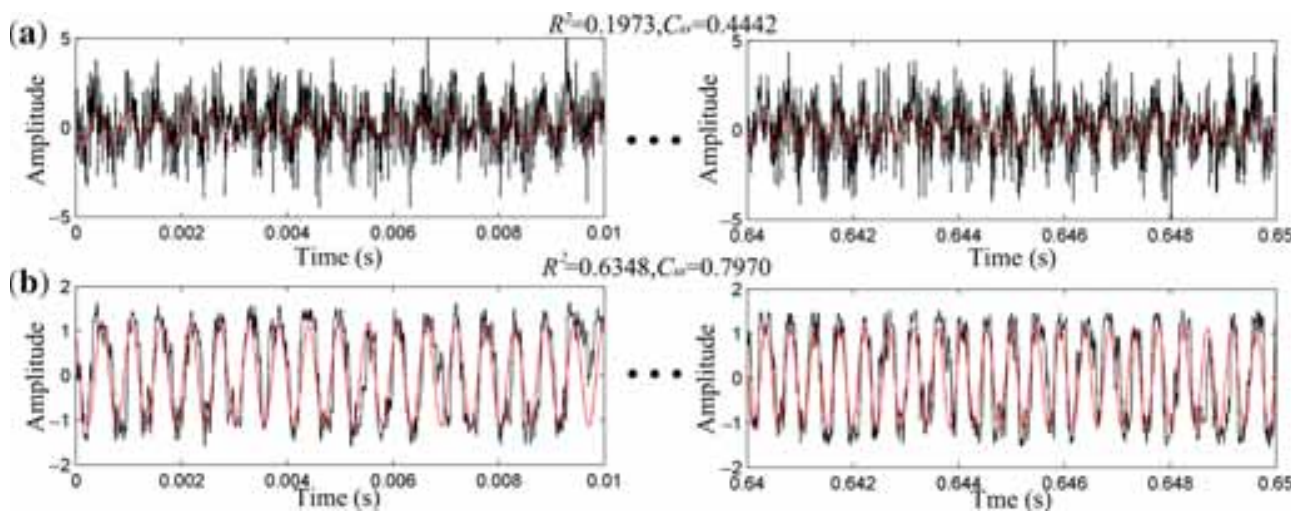

Figure 7. Doppler acceleration input signal with noise and its optimal output waveform: (a) original input signal with noise of $-3 \mathrm{~dB}$, and (b) optimal output waveform. 

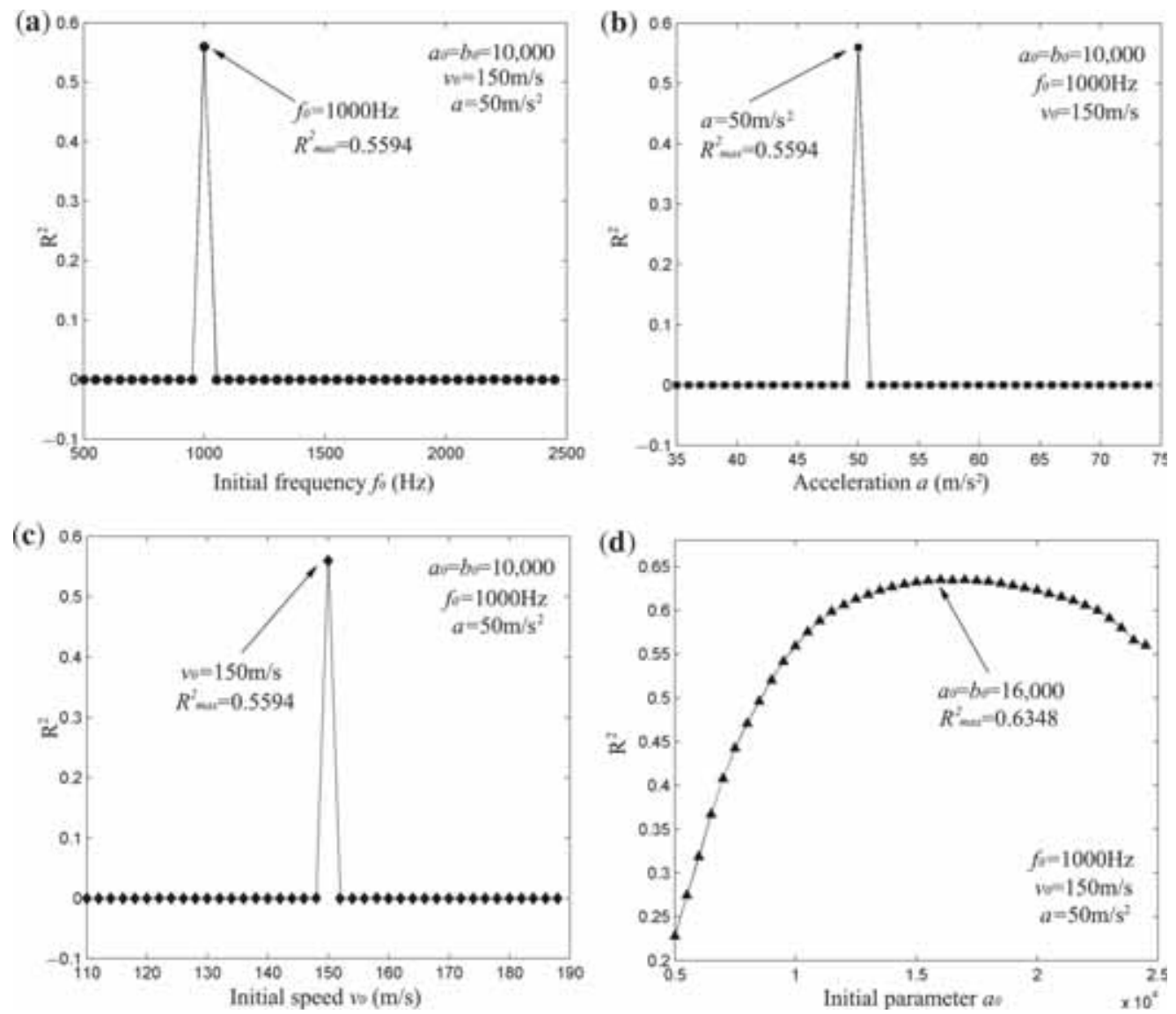

Figure 8. Doppler acceleration signal output $R^{2}$ with variation of parameters for VPNSR, (a) $R^{2}$ with variation of initial frequency $f_{0}$, (b) $R^{2}$ with variation of acceleration $a$, (c) $R^{2}$ with variation of initial speed $v_{0}$, and (d) $R^{2}$ with variation of system parameter $a_{0}$.

preset system parameters $a_{0}=b_{0}=10,000$. Use $s^{\prime}(t)=$ $A^{\prime} \cos \left(\frac{2 \pi c \hat{f}_{0}}{\hat{a}} \ln \left(c-\hat{v}_{0}-\hat{a} t\right)+\varphi^{\prime}\right)$ to fit the output signal $x(t)$ of each set of $\hat{f}_{0}, \hat{v}_{0}, \hat{a}$ and take the $R^{2}\left(\hat{f}_{0}, \hat{v}_{0}, \hat{a}\right)$ as the output criterion. Figure 8 shows us the estimation results where the one-dimensional search is exhibited for better illustration. In figures 8(a)-(c), extremely high peaks are found for each signal parameter with the other two fixed at their true values. As the signal length here is much longer than that of the LMF, a small error of parameter estimation will lead to a large reduction of output fitness coefficient. In fact, a three-dimensional search is conducted here to obtain the estimates $v_{0}=150 \mathrm{~m} / \mathrm{s}, f_{0}=1000 \mathrm{~Hz}$ and $a=50 \mathrm{~m} / \mathrm{s}^{2}$. Then with the right signal parameters, change $a_{0}\left(b_{0}\right)$ from 5000 to 24,500 with varying step of 500. Substituting every set of parameters into Eq. (9) and solving it with normalization, the output $R^{2}$ changing with different $a_{0}\left(b_{0}\right)$ are shown as figure 8(d). Apparently, when set $a_{0}=b_{0}=16,000$, the output $R^{2}$ gets to the maximal value of 0.6348. Till now, all the parameters are detected or optimized successfully and what needs to be obtained is the final output indicated in figure $7(\mathrm{~b})$. The results show us that both $R^{2}$ and $C_{s x}$ get highly improved to the value of 0.6348 and 0.7970 , which means the signal is recovered well with the signal parameters detected successfully. In its waveform, the more distinct fluctuation signal is found than the input one with noise eliminated, which can be made out even in time domain.

\section{Analysis of system output performance}

In last section, we have introduced the whole detection and recovery procedure of time-varying signal suffered from background noise referring to the LFM signal and Doppler signal with acceleration. The results show us the availability and superiority of the proposed VPNSR in such application area. The output criteria get highly improved by the system and it affords us the satisfying signal for the further processing. The system performance of different input signal was revealed and the signal parameters influence on its output was shown with the LFM signal by numerical simulation. The inner principle of the system's outstanding property is explained. 


\subsection{Influence of initial frequency}

In the expression of a LFM signal, there are two main parameters that affect the signal features which are initial frequency and modulated rate. Firstly, fix the modulated rate at $k=10,000 \mathrm{~Hz} / \mathrm{s}$ while change $f_{0}$ from $10 \mathrm{~Hz}$ to $10,000 \mathrm{~Hz}$ logarithmically. All the other variables are same as section 4.1 with $\varphi=0, A=1, f_{s}=200 \mathrm{kHz}$, sampling time $T=0.1 \mathrm{~s}$ and input SNR $=-3 \mathrm{~dB}$. Then for each $f_{0}$, there is a certain input signal. First of all, calculate the input fitness coefficient $R_{0}^{2}$ and cross-correlation coefficient $C_{s x 0}$. Then traditional SR system is taken to process the different signals with optimal output criteria of $R_{1}^{2}$ and $C_{s x 1}$. Finally the signals are processed by the proposed VPNSR with optimal output criteria of $R_{2}^{2}$ and $C_{s x 2}$. Figure 9 shows us the input and output results with different initial frequency where the red line indicates the VPNSR, blue line means the traditional SR and black one shows the input results.

In the two graphs of $R^{2}$ and $C_{s x}$, a similar variation tendency is found. When compared to the input signal, both of the two systems can improve the output quality with noise suppressed and original signal recovered. And with the increasing of $f_{0}$, the two criteria both decrease which coincide with the SR theory well. A high initial frequency will lead to a high driving frequency and a bad performance of the system. But the two systems have the different performance with different $f_{0}$. When $f_{0}$ is large enough, the two models have the almost same output but the VPNSR owns a better output than the traditional one under a low level of $f_{0}$. Besides, the smaller $f_{0}$, the more evident superiority VPNSR possesses. This is easy to understand via the frequency band. The normalization is taken to reduce the driving frequency by $a$ or $a(t)$. With a fixed $k$ and the same noise level, a smaller $f_{0}$ will result in a smaller $a$ in traditional SR and inevitably a wider frequency
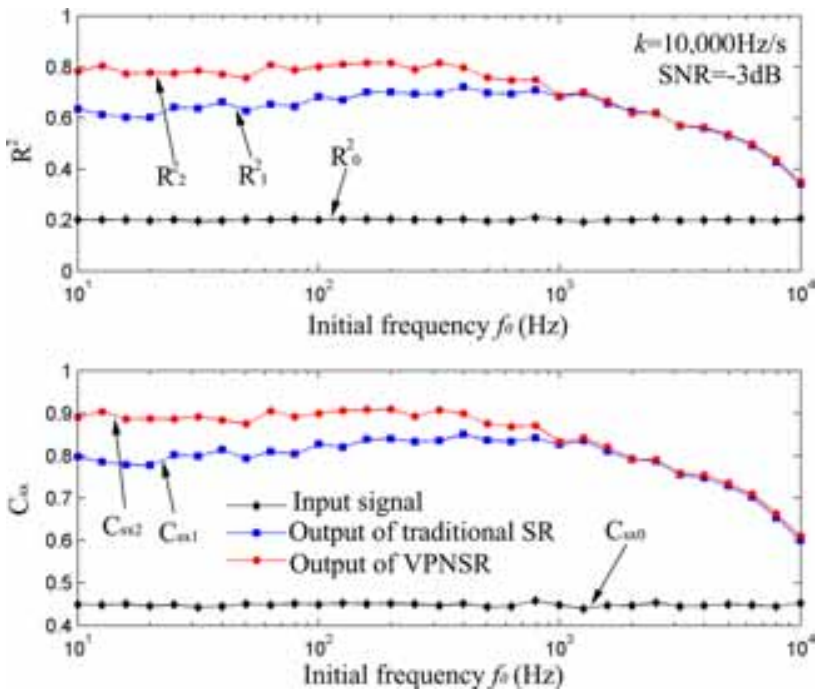

Figure 9. Input and output results of different systems with variation of initial frequency $f_{0}$. band relatively of $\left[\frac{f_{0}}{a}, \frac{f_{0}+k T}{a}\right]$. The width of frequency band is an awful factor to the traditional SR and its output gets worse and worse. But to VPNSR the limitation is effectively avoided and when initial frequency decreases, system even performs better on the contrary.

\subsection{Influence of modulated rate}

Later, aiming to find the influence of modulated rate $k$, we fix $f_{0}=100 \mathrm{~Hz}$. All the other input parameters are same as before. Meanwhile, vary $k$ from $10 \mathrm{~Hz} / \mathrm{s}$ to $10,000 \mathrm{~Hz} / \mathrm{s}$ logarithmically and calculate the different output for each $k$ as shown in figure 10. The two figures indicate the results of fitness coefficient $R^{2}$ and cross-correlation coefficient $C_{s x}$ separately where the red line means the VPNSR, blue line means the traditional SR and black one is the input results.

In the figures, both of the two criteria are far more improved when compared to the input signal while they decrease with the increasing of modulated rate $k$. But the descend rate of the VPNSR is much slower than the traditional SR which means a larger $k$ will result in a better performance of the proposed system relatively. This can be also explained by the driving frequency band accessibly. With the same parameters except for $k$, a larger modulated rate will lead to a wider frequency band. The input signal contains more components of different frequency in this case. Then the traditional SR will not offer a satisfying output and cannot recover the original signal in the whole band, but not for the VPNSR. It overcomes the limitation by concentrating the frequency into one point and realizes the SR effect of time-varying signal. So the proposed system highly improves the ability of an SR system to deal with wide-band time-varying signal.
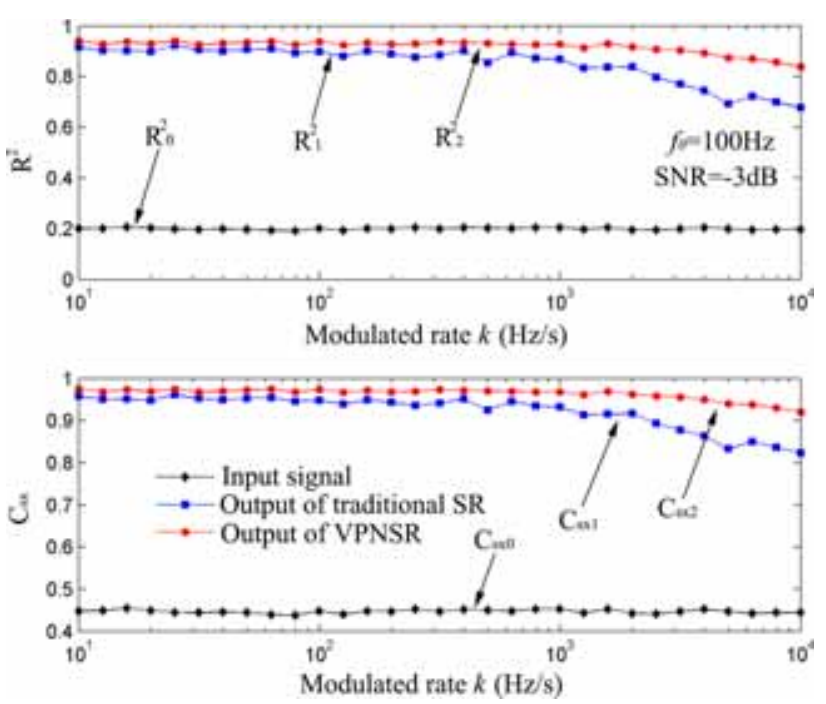

Figure 10. Input and output results of different systems with variation of modulated rate $k$. 

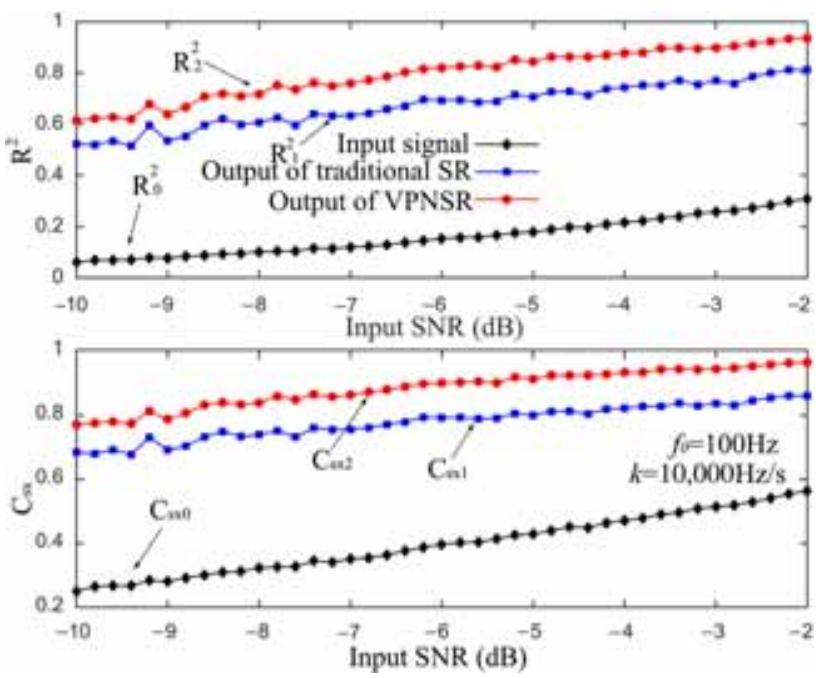

Figure 11. Input and output results of different systems with variation of input SNR.

\subsection{Influence of input SNR}

In the last two subsections, we discussed the effect of signal parameters on system performance. Now we consider the influence of input SNR. The initial frequency and chirp rate are fixed at $100 \mathrm{~Hz}$ and $10,000 \mathrm{~Hz} / \mathrm{s}$. All the other input parameters are same as in section 4.1 except for the input SNR which varies from $-2 \mathrm{~dB}$ to $-10 \mathrm{~dB}$ added by awgn in Matlab. Different output results of fitness coefficient $R^{2}$ and cross-correlation coefficient $C_{s x}$ are shown in figure 11 . In this case, the red lines mean the VPNSR output, blue lines indicate the traditional SR output and black ones are the input results similarly. Simulation results reveal that both of the two criteria exhibit a high promotion of the VPNSR than the traditional SR system as well as the input signal in the whole range of different input SNR. Although the criteria get lower with the SNR increasing, the proposed system still performs well with a high input SNR and provides a more satisfying output than the traditional SR system.

The above studies indicate that the VPNSR proposed in this paper performs well in the denoising of a time-varying signal like LFM and acceleration Doppler as well as recovering of them. The superiority gets more obvious for input LFM signal with a low initial frequency and high modulated rate which means the wider-band time-varying signal in fact than the traditional SR. The system possesses much strong adaptability for this kind of signal which can be hoped to be widely used in some relative areas.

\section{Conclusions}

This paper proposed a VPNSR system aiming at timevarying signal with certain type. The system can be utilized to detect the signal parameters and recover the original signal ruined by background noise. Fitness coefficient is regarded as the index to estimate the signal parameters. When they are confirmed successfully, the $R^{2}$ is employed again to search for the optimal system parameters. Substituting the two pairs of parameters into Eq. (9) we get the optimal output signal with noise eliminated and original signal recovered. The simulating results show us that the proposed system can perform much well in the wide-band signal detection and recovery and possess the advantages as follows.

1. The system combines the normalization of SR which releases the limitation of small parameters resulting from the adiabatic approximation theory. Input signal with high driving frequency can also be recovered successfully by the system from the simulation results.

2. The time-varying system parameters $a(t)$ and $b(t)$ are taken into consideration which are different from the traditional SR. The improvement enables us to process the time-varying signal with wider frequency band. This provides us the new approach and idea to deal with the aperiodic signal like LFM or accelerated Doppler signal.

3. According to the simulating results in this paper, the proposed method performs well especially for signal with small initial frequency and fast frequency fluctuation rate. In other words, when the signal to be processed has a wider frequency band, the traditional SR will perform worse but the more obvious superiority VPNSR will possess which means it has the excellent availability and adaptability to the wide-band signal.

As all the merits, the proposed system can be hoped to be widely used in signal processing or other areas like radar communication. But there are still several challenges need to overcome one of which is that the signal type needs to be foreknown. This indicates that the nature of time-variation of the system parameters depends on the nature of the signal during the realization of the method of the doublewell potential system with time-varying parameters. In this case, the system needs to be tuned referring to the nature of input time-varying signal and adapted to the input signal. The computational efficiency of multi-dimensional searching and curve fitting algorithm as well as the searching accuracy need to be improved. In our future work, we will focus on the improvement of algorithm efficiency for the unknown type signal and applying it to the practical signal and engineering application.

\section{Abbreviations}

SNR signal-to-noise ratio

SR stochastic resonance

LFM frequency modulated signal

NLFM nonlinear frequency modulated

ASR aperiodic stochastic resonance 
VPNSR varying parameters and normalization stochastic resonance

KR Kramers rate

\section{Acknowledgements}

This work was supported in part by the National Natural Science Foundation of China with the Grant Nos. 51475441 and 11274300 . Besides, the authors also would like to thank the anonymous reviewers for their valuable comments and suggestions.

\section{Reference}

[1] Benzi R, Sutera A and Vulpiani A 1981 The mechanism of stochastic resonance. J. Phys. A-Math. General 14: L453L457

[2] Gammaitoni L, Hanggi P, Jung P and Marchesoni F 1998 Stochastic resonance. Rev. Modern Phys. 70: 223-287

[3] Dykman M I and McClintock P V E 1998 What can stochastic resonance do? Nature 391: 344-344

[4] Gammaitoni L, Marchesoni F, Menichellasaetta E and Santucci S 1989 Stochastic resonance in bistable systems. Phys. Rev. Lett. 62: 349-352

[5] Chen H and Varshney P K 2008 Theory of the stochastic resonance effect in signal detection-Part II: Variable detectors. IEEE Trans. Signal Process. 56: 5031-5041

[6] Jung P and Hanggi P 1991 Amplification of small signals via stochastic resonance. Phys. Rev. A 44: 8032-8042

[7] Leng Y G, Wang T Y, Guo Y, Xu Y G and Fan S B 2007 Engineering signal processing based on bistable stochastic resonance. Mech. Syst. Signal Process. 21: 138-150

[8] Lu S, He Q and Kong F 2015 Effects of underdamped stepvarying second-order stochastic resonance for weak signal detection. Digital Signal Process. 36: 93-103

[9] Zozor S and Amblard P O 2002 On the use of stochastic resonance in sine detection. Signal Process. 82: 353-367

[10] He Q B, Wang J, Liu Y B, Dai D Y and Kong F R 2012 Multiscale noise tuning of stochastic resonance for enhanced fault diagnosis in rotating machines. Mech. Syst. Signal Process. 28: 443-457

[11] Lei Y G, Lin J, Han D and He Z J 2014 An enhanced stochastic resonance method for weak feature extraction from vibration signals in bearing fault detection. Proceedings of the Institution of Mechanical Engineers Part C-J. Mech. Eng. Sci. 228: 815-827

[12] Li J M, Chen X F and He Z J 2013 Multi-stable stochastic resonance and its application research on mechanical fault diagnosis. J. Sound Vib. 332: 5999-6015

[13] Tan J Y, Chen X F, Wang J X, Chen H X, Cao H R and Zi Y Y 2009 Study of frequency-shifted and re-scaling stochastic resonance and its application to fault diagnosis. Mech. Syst. Signal Process. 23: 811-822

[14] Gammaitoni L, Neri I and Vocca H 2009 Nonlinear oscillators for vibration energy harvesting. Appl. Phys. Lett. 94: 164102(1)-164102(3)

[15] Harne R L and Wang K W 2013 A review of the recent research on vibration energy harvesting via bistable systems. Smart Mater. Struct. 22: 023001(1)-023001(12)

[16] McInnes C R, Gorman D G and Cartmell M P 2008 Enhanced vibrational energy harvesting using nonlinear stochastic resonance. J. Sound Vib. 318: 655-662

[17] Singh A K and Saxena R 2013 Doppler estimation from echo signal using FRFT. Wirel. Pers. Commun. 72: 405-413

[18] Wang M S, Chan A K and Chui C K 1998 Linear frequencymodulated signal detection using radon-ambiguity transform. IEEE Trans. Signal Process. 46: 571-586

[19] Wang P, Li H B and Himed B 2008 Parameter estimation of linear frequency-modulated signals using integrated cubic phase function, 2008 42nd Asilomar Conference on Signals, Systems and Computers, vol 1-4, pp. 487-491

[20] Collins J J, Chow C C and Imhoff T T 1995 Aperiodic stochastic resonance in excitable systems. Phys. Rev. E 52: R3321-R3324

[21] Collins J J, Chow C C, Capela A C and Imhoff T T 1996 Aperiodic stochastic resonance. Phys. Rev. E 54: 5575-5584

[22] Hu G, Gong D C, Wen X D, Yang C Y, Qing G R and Li R 1992 Stochastic resonance in a nonlinear-system driven by an aperiodic force. Phys. Rev. A 46: 3250-3254

[23] Eichwald C and Walleczek J 1997 Aperiodic stochastic resonance with chaotic input signals in excitable systems. Phys. Rev. E 55: R6315-R6318

[24] Yang D X and Hu N Q 2004 Detection of weak aperiodic shock, signal based on stochastic resonance. In: Proceedings of the Third International Symposium on Instrumentation Science and Technology, vol 1, pp. 209-21325

[25] Peng H, Zhong S C, Tu Z and Ma H 2013 Stochastic resonance of over-damped bistable system driven by chirp signal and Gaussian white noise. Acta Physica Sinica 62: 080501(1)-080501(6)

[26] Hu N Q, Chen M and Wen X S 2003 The application of stochastic resonance theory for early detecting rub-impact fault of rotor system. Mech. Syst. Signal Process. 17: 883-895

[27] Øksendal B 2003 Stochastic differential equations. In: Stochastic differential equations, ed: Springer Berlin Heidelberg, pp. 65-84

[28] Leng Y G, Yong S L, Wang T Y and Yan G 2006 Numerical analysis and engineering application of large parameter stochastic resonance. J. Sound Vib. 292: 788-801

[29] Park K, Lai Y C, Liu Z H and Nachman A 2004 Aperiodic stochastic resonance and phase synchronization. Phys. Lett. A 326: 391-396

[30] Morse P K and Ingard K U 1968 Theoretical acoustics (section 2). New York: Princeton University Press 